\title{
The Upper Complement Connected Monophonic Number of a Graph
}

\author{
F. Merlin Sugirtha, P.Arul Paul Sudhakar, S.Robinson Chellathurai
}

Abstract: For a connected graph $G=(V, E)$, a monophonic set ${ }^{M}$ of ${ }^{G}$ is said to be a complement connected monophonic set if $M=V$ or the subgraph $\langle V-M\rangle$ is connected. The minimum cardinality of a complement connected monophonic set of ${ }^{G}$ is the complement connected monophonic number of $G$ and is denoted by $m_{c e}(G)$. $A$ complement connected monophonic set ${ }^{M}$ in a connected graph $G$ is called a minimal complement connected monophonic set if no proper subset of ${ }^{M}$ is a complement connected monophonic set of ${ }^{G}$. The upper complement connected monophonic number $m_{c e}^{+}(G)$ of $G$ is the maximum cardinality of a minimal complement connected monophonic set of ${ }^{G}$. Some general properties under this concept are studied. The upper complement connected monophonic number of some standard graphs are determined. Some of its general properties are studied. It is shown that for any positive integers $2 \leq a \leq b$, there exists $a$ connected graph ${ }^{G}$ such that $\left.m_{C C}{ }^{G}\right)=$ a and $\left.m_{C C(}^{+} G\right)=b$.

Key Words: monophonic path, complement connected monophonic number, upper complement connected monophonic number.

\section{INTRODUCTION}

We consider finite graphs with neither loops nor multiple edges. For any graph $G$, we denote the vertex set by $V(G)$ and

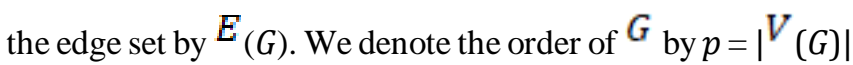
and the size by $q=\left.\right|^{E}(G) \mid$. The minimum and maximum degrees of a graph ${ }^{G}$ are denoted by ${ }^{\delta}={ }^{\delta}(G)$ and $\Delta=\Delta(G)$ respectively. The subgraph induced by a set $M_{\text {of vertices of a }}$ graph $G$ is denoted by $\langle M\rangle$ with $(\langle M\rangle)=M$ and $\mathrm{E}(\langle M\rangle)$ $=\{u v \in E(G): u, v \in M\}$. For basic graph theoretic terminology we refer to [1]. A chord of a path $u 0, u 1, u 2, \ldots, u n$ is an edge ${ }^{u_{i} u_{j}}$ with $j \geq i+2$. An u$\mathrm{v}$ path is called a monophonic path if it is a chordless path.

Revised Manuscript Received on July 08, 2019.

F. Merlin Sugirtha ${ }^{1}$ Register Number-12447, Scott Christian College, Nagercoil-629 003, India.

P.Arul Paul Sudhakar Assistant Professor, Department of Mathematics,Rani Anna Government Arts College (W), Tirunelveli-627 012, India.

S.Robinson Chellathurai, Associate Professor, Department of Mathematics, Scott Christian College, Nagercoil-629 003, India.

The monophonic number $\mathrm{m}\left({ }^{G}\right)$ of $G$ is the minimum order of its monophonic sets and any monophonic set of order $\mathrm{m}\left({ }^{G}\right)$ is a minimum monophonic set or simply a m-set of $G$. A monophonic set ${ }^{M}$ of a connected graph ${ }^{G}$ is said to be a complement connected monophonic set if $M=V$ or the subgraph $\langle V-M\rangle$ is connected. The minimum cardinality of a complement connected monophonic set of ${ }^{G}$ is the complement connected monophonic number of $G$ and is denoted by $m_{c c}(G)$. A complement connected monophonic

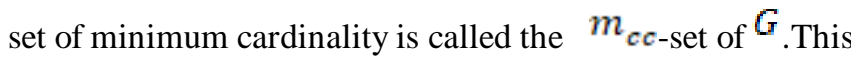
concept is introduced in [6]. In this paper, we study the upper complement connected monophonic number of a connected graph.

The following theorem is used in the sequel.

Theorem 1.1[6] Every complement connected monophonic set of $G$ contains its extreme vertices.

\section{THE UPPER COMPLEMENT CONNECTED MONOPHONIC NUMBER OF A GRAPH}

Definition 2.1. A complement connected monophonic set $M$ in a connected graph ${ }^{G}$ is called a minimal complement connected monophonic set if no proper subset of ${ }^{M}$ is a complement connected monophonic set of ${ }^{G}$.The upper complement connected monophonic number $m_{c e}^{+}(G)$ of $G$ is the maximum cardinality of a minimal complement connected monophonic set of ${ }^{G}$.

Example 2.2. For the graph $\mathrm{G}$ given in Figure 2.1, $\mathrm{M}=$ $\left\{v_{1}, v_{3}\right\}$ is $m_{\text {-set of }} G$. $M_{1}=\left\{v_{1}, v_{2}, v_{5}, v_{6}\right\}, M_{2}=$ $\left\{v_{2}, v_{3}, v_{4}, v_{6}\right\}$ are the only two $m_{c E \text {-sets so that }}$ $m_{c e}(G)=4$. Also M3 $=\left\{\mathrm{v} 1, \mathrm{v} 3, \mathrm{v} 4, v_{5}, v_{6}\right\}$ and $M_{4}=\left\{v_{1}, v_{2}, v_{3}, v_{4}, v_{5}\right\}$ are minimal complement connected monophonic sets and so $m_{c E}^{+}(G)_{\geq} 5$. Since 


\section{The Upper Complement Connected Monophonic Number of a Graph}

$|V(G)|=6$, it follows that $m_{c \in}^{+}(G)=5$.

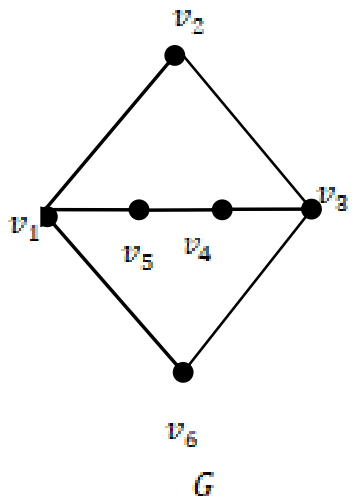

Figure

Remark 2.3. Every minimum complement connected monophonic set of $G$ is a minimal complement connected monophonic set of $\mathrm{G}$ and the converse is not true. For the graph $\mathrm{G}$ given in Figure 2.1, M4= $\left\{\mathrm{v} 1, v_{2}, \mathrm{v} 3, v_{4,}^{v_{v}} 5\right\}$ is a minimal complement connected monophonic set but not a minimum monophonic set of $\mathrm{G}$.

Observation 2.4

(i) Each extreme vertex of $G$ belongs to every complement connected monophonic set of $G$.

(ii) Let ${ }^{G}$ be a connected graph with cut-vertices and $S_{\text {be a complement connected }}$ monophonic set of $G$. If ${ }^{v}$ is a cut-vertex of $G$, then every component of $G-v$ contains an element of $S$.

(iii) For any connected graph ${ }^{G}$, no cut-vertex of $G$ belongs to any minimal complement connected monophonic set of $G$.

Corollary 2.5. For any non-trivial tree, the complement connected monophonic number $\quad m_{c c}^{+}(T)=m(T)=k$,

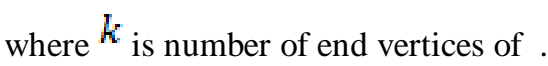

Proof: This follows from Observations 2.4 (i) and (iii).

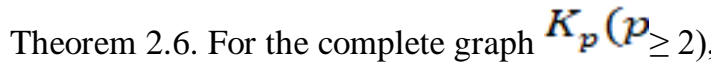
$\left.\left.m_{c c+(} K_{p}\right)=m_{(} K_{p}\right)=p$.

Proof: Since every vertex of the complete graph, $K p(p \geq 2)$ is an extreme vertex, the vertex set of $\mathrm{Kp}$ is the unique complement connected monophonic set of Kp. Thus $m_{C C}^{+}(\mathrm{Kp})=\mathrm{m}(\mathrm{Kp})=\mathrm{p}$.

Theorem 2.7.For a cycle ${ }^{G}=\mathrm{Cp}(\mathrm{p} \geq 4), m_{c c+(\mathrm{G})}=3$. Proof: Let $v_{1}, v_{2}, v_{3}, \ldots v_{p}, v_{1}$ be the cycle $C_{p}$.

$$
\begin{gathered}
\text { Let } \quad M=\left\{v_{i}, v_{i+1}, v_{i+2}\right\}, 1 \leq i \leq p-2 \\
M^{r}=\left\{v_{p-1}, v_{p}, v_{1}\right\} \text { and } M^{\mathrm{I}}=\left\{v_{p}, v_{1}, v_{2}\right\} .
\end{gathered} \text {, }
$$
Then $M_{i}(1 \leq i \leq p-2), M^{r}$ and $M^{\mathrm{m}}$ are $m_{c c-s e t s}$ of ${ }^{G}$ so that $m_{c c}(G)=3$. We prove that $m_{c c+(G)}=3$. If not, let $m_{c c+(\mathrm{G})} \geq 4$. Then there exists a minimal complement connected monophonic set ${ }^{S}$ such that $|\mathrm{S}| \geq 4$. Then either $\langle V-S\rangle$ is connected or any one of $M_{i}(1 \leq i \leq p-2), M^{T}, M^{\mathrm{m}}$ is a subset of ${ }^{S}$ and so $S$ is not a minimal complement connected monophonic set of $\mathrm{G}$, which is a contradiction. Therefore, $m_{c c+(\mathrm{G})}=3$.

Theorem 2.8. For the complete bipartite graph $G=K_{m_{s} n}$,

$m_{c c}^{+}(G)=\left\{\begin{array}{c}n, \text { if } m=1, n \geq 2 \\ 3, \text { if } m=n=2 \\ 4, \text { if } 2<m \leq n\end{array}\right.$

Proof: If $m=1, n \geq 2$, then by corollary 2.5 , $m_{c e}^{+}(G)=n$. If $m=n=2$, then by

Theorem 2.7, $m_{c c}^{+}(G)=3$. So let $2<m \leq n$. Let $U=\left\{u_{1}, u_{2}, \ldots, u_{m}\right\}$ and

$V=\left\{v_{1}, v_{2}, \ldots, v_{n}\right\}$ be the two bipartite sets of ${ }^{G}$. Let $u, x \in U$, and $v, w \in V$. Then

$S=\{u, x, v, w\}$ is a minimal complement connected monophonic set of ${ }^{G}$ so that

$m_{c c+(\mathrm{G})} \geq_{4}$. We prove that $m_{c c+(\mathrm{G})=4}$. Suppose that $m_{c c+(\mathrm{G})} \geq 5$. Then there exists a complement connected monophonic set $M$ 
such that $|M| \geq 5$. Then $S \subset M$.so that $M$ is not a complement connected monophonic set which is a contradiction.

Therefore $m_{c c+(\mathrm{G})=4}$.

Theorem 2.9. For a

$$
G=W_{p}=K_{1}+C_{p-1}, m_{c c}^{+}(G)=2 .
$$

Proof: Let $x, y$ be two non adjacent vertices of ${ }^{G}$. Then $S=\{x, y\}$ is a minimal

complement connected monophonic set of $G$ and so $m_{c c}^{+}(G) \geq 2$. We prove that

$m_{c c}^{+}(G)=2$. Suppose that $m_{c c}^{+}(G) \geq 3$. Then there exists a complement connected monophonic set $M_{\text {such that }}|M| \geq 3$. Then $S \subset M$ so that $M$ is not a complement connected monophonic set which is a contradiction.

Therefore $m_{c c}^{+}(G)=2$

Theorem 2.10. For a connected graph ${ }^{G}, 2 \leq m_{(\mathrm{G})} \leq m_{c c+}$ $(\mathrm{G}) \leq \mathrm{p}$.

Proof: Any complement connected monophonic set needs at least two vertices and so $\mathrm{m}(\mathrm{G}) \geq 2$. Since every minimal complement connected monophonic set is a complement connected monophonic set, $\left.\mathrm{m}\left({ }^{G}\right) \leq m_{c c+}{ }^{G}\right)$. Also, since $\mathrm{V}\left({ }^{G}\right)$ is a complement connected monophonic set of $G$, it is

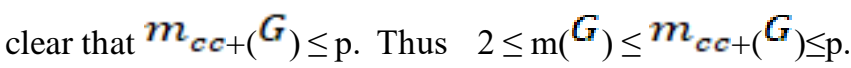

Theorem 2.11. For a connected graph $G, m_{c c}(G)=p$ if and only if $m_{c c+}(G)=$.

Proof: If $m_{c c}(G)=p$, then by Theorem $2.10, m_{c c+}$ $\left({ }^{G}\right)=$ p. Conversely, let $m_{c c+}(G)=$. Then $S=V(G)$ is the unique minimal complement connected monophonic set of $G$. Since no proper subset of ${ }^{S}$ is a complement connected monophonic set of $G, S$ is the unique minimum complement connected monophonic set of $G$ so that $m_{c c}(G)=p$.

Theorem 2.12.For a connected graph $G$ if $m_{c c}(G)=p_{-1, \text { then }} m_{c c+}\left({ }^{G}\right)=\mathrm{p}-1$.
Proof: Let $m_{c c}(G)=p_{-1}$. Then by Theorem 2.10,

$$
m_{c c+}(G) \text { is either } p \text { or } p-1 \text {. If }
$$

$m_{c c+}(G)=p$, then by Theorem $2.11, m_{c c}(G)=p$, which is a contradiction. Therefore $m_{c c+}(G)=\mathrm{p}-1$.

Remark 2.13. The converse of Theorem 2.12 need not be true.

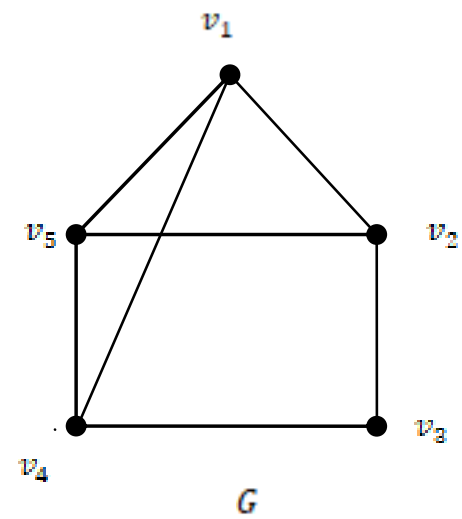

Figure 2.2

For the graph ${ }^{G}$ given in Figure 2.2, $S=\left\{v_{3}, v_{5}\right\}$ is a $m_{\overline{C E} \text {-set of }}{ }^{G}$ so that

$m_{c e}(G)=2<p-1$. Also $S=\left\{v_{1}, v_{2}, v_{4}, v_{5}\right\}$

is a minimal complement connected monophonic set of ${ }^{G}$ so that $m_{c c}+(G)=4=p-1$. But $m_{C E}(G)=2 \neq p-1$.In view of Theorem 2.10, we have the following realization result.

Theorem 2.14. For any positive integers $2 \leq \mathrm{a} \leq \mathrm{b}$ and $b>a+2$, there exists a connected graph ${ }^{G}$ such that $m_{C \mathcal{C C}}\left({ }^{G}\right)=\mathrm{a}$ and $m_{\operatorname{cc}+\left({ }^{G}\right)}=\mathrm{b}$.

Proof: Let ${ }^{G}$ be a graph given in Figure 2.3 obtained from the path on three vertices $\mathrm{P}:^{x, y}, \boldsymbol{z}$ by adding the new vertices $v_{1}, v_{2}, \ldots, v_{b-a+2}$ and $z_{1}, z_{2}, \ldots, z_{a-2}$ and joining vi to $\boldsymbol{v}_{i+1}(1 \leq \mathrm{j} \leq \mathrm{b}-\mathrm{a}+1)$, and also joining each $\mathrm{zi}(1 \leq \mathrm{i} \leq \mathrm{a}-2)$ with $\mathrm{y}$ and join $\boldsymbol{y}_{\text {with }} \boldsymbol{v}_{1}$. First we show that $m_{C C}(G)=$ a. Let $\mathrm{M}$ be a complement connected monophonic set of $\mathrm{G}$ and let $\mathrm{Z}=\left\{z_{1}, z_{2}, \ldots, z_{a-2}\right\}$. By Theorem 1.1, $\mathrm{Z} \subseteq \mathrm{M}$.. It is easily seen that $\mathrm{Z}$ is not a complement connected monophonic set of $\mathrm{G}$ and so $m_{c c}(G) \geq a-1$ 


\section{The Upper Complement Connected Monophonic Number of a Graph}

$Z \cup\{u\}, u \notin Z$ is not a complement connected monophonic set of $G$ and so $m_{c c}(G) \geq a$. Now $S=Z \cup\{x, y\}$ is a complement connected monophonic set of G so that $m_{c c}(G)=a$. Next we show

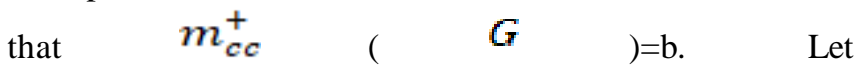
$\mathrm{M} 1=\mathrm{ZU}\left\{v_{1}, v_{2}, \ldots, v_{b-a+2}\right\}$.Then $M_{1}$ is a complement connected monophonic set of $G$. We prove that $M_{1}$ is a minimal complement connected monophonic set of G.

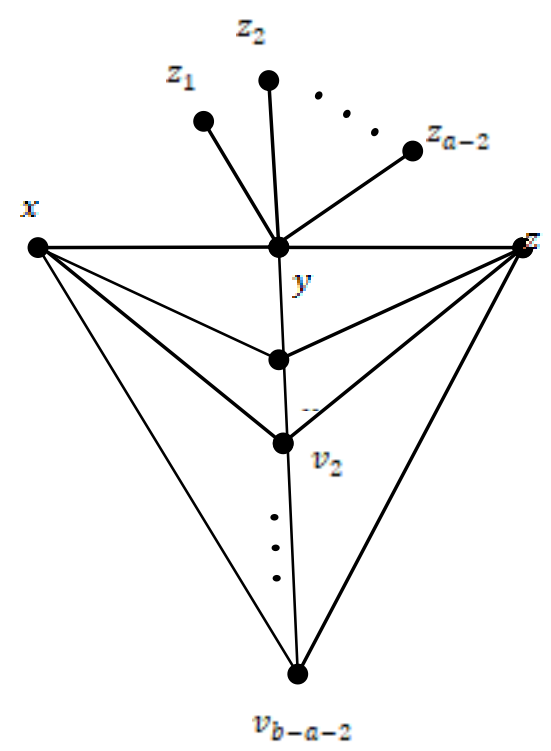

G

Figure 2.3

If M1 is not a minimal complement connected monophonic set of ${ }^{G}$, then there is a proper subset T of M1suchthat T is a complement connected monophonic set of $G$.Then there

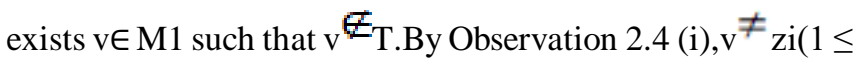
$\mathrm{i} \leq \mathrm{a}-2)$. Therefore $\mathrm{v}=\mathrm{vi}$ for some $\mathrm{i}(1 \leq \mathrm{i} \leq \mathrm{b}-\mathrm{a}+2)$. Then $v$ does not lie on any monophonic path joining some pair of vertices of $\mathrm{T}$ and so $\mathrm{T}$ is not a complement connected monophonic set of ${ }^{G}$, which is a contradiction. Therefore M1 is a minimal complement connected monophonic set of $G$ and so $m_{C E}^{+}\left({ }^{G}\right) \geq \mathrm{b}$. We show that $m_{c \mathcal{C}}^{+}(G)=b$. Let $\mathrm{T}$ 'be a minimal complement connected monophonic set of $G$ with $\left|T^{I}\right| \geq b+1$. By Observation 2.4 (i), ${ }^{Z} \subseteq \mathrm{T}^{\prime}$. Since

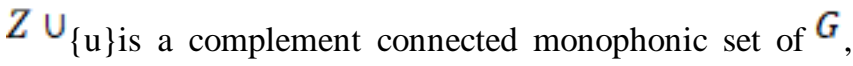
$\mathrm{u}^{\mathbb{E}} \mathrm{T}^{\prime}$. Since M1 is a complement connected monophonic set of ${ }^{G}$, there exists at least one vi such that vi ${ }^{\mathbb{E}_{\mathrm{T}}}$. Without loss

of generality let us assume that ${ }^{\mathrm{E}}{ }^{\mathbb{E}} \mathrm{T}^{\prime}$. Since $\left.\left.\right|^{T^{\natural} \mid}\right|_{\geq \mathrm{b}+2}$, then $y$ must belong to $T^{\prime}$. Now it is clear that $\mathrm{v} 1$ does not lie on a complement connected monophonic path joining a pair of vertices of $\mathrm{T}$ ', it follows that $\mathrm{T}$ 'is not a complement connected monophonic set of ${ }^{G}$, which is a contradiction. Therefore $m_{c c+}(G)=$ b.

\section{REFERENCES}

1. F.Buckley and F.Harary, Distance in Graphs, Addition Wesley, Redwood City, CA, 1990.

2. Esamel M. paluga, Sergio R. Canoy, Jr. Monophonic numbers of the join and Composition of connected graphs, Discrete Mathematics, 307 (2007), $1146-1154$

3. J. John and S. Panchali, The Upper Monophonic Number of a graph, International J. math. Combin. 4(2010), 46-52.

4. J. John, P. Arul Paul Sudhahar and A. Vijayan, 'The Connected Monophonic Number of a graph', International Journal of Combinatorial Graph Theory and Applications, Vol.,No.1, (January-June 2012), pp 83-90.

5. J. John and P. Arul Paul Sudhahar on The Edge Monophohic Number of a Graph, Filomat 26:6(2012), 1081-1089.

6. F. Merlin Sugirtha, P. Arul Paul Sudhahar and S. Robinson Chellathurai, On The Complement Connected Monophonic Number of a Graph, International Journal of Scientific Research and Review, ISSN NO:2279-543X,Vol 7,Issue 11,2018, 225-231.

\section{AUTHORS PROFILE}

F. Merlin Sugirtha ${ }^{1}$ Register Number-12447, Scott Christian College, Nagercoil-629 003, India.

P.Arul Paul Sudhakar Assistant Professor, Department of Mathematics,Rani Anna Government Arts College (W), Tirunelveli-627 012, India.

S.Robinson Chellathurai, Associate Professor, Department of Mathematics, Scott Christian College, Nagercoil-629 003, India. 